\title{
(2) OPEN ACCESS \\ Prevalence, incidence and survival of heart failure: a systematic review
}

\author{
Sophia Emmons-Bell, ${ }^{1}$ Catherine Johnson, ${ }^{1}$ Gregory Roth $๑^{1,2}$
}

\begin{abstract}
- Additional supplemental material is published online only. To view, please visit the journal online (http://dx.doi. org/10.1136/heartjnl-2021320131).
\end{abstract}

'Institute for Health Metrics and Evaluation, University of Washington, Seattle, Washington, USA 2Division of Cardiology, Department of Medicine, University of Washington, Seattle, Washington, USA

\section{Correspondence to Dr Gregory Roth, Division of Cardiology, Department of Medicine, University of Washington, Seattle, Washington, USA rothg@uw.edu}

Received 12 August 2021 Accepted 22 November 2021

Check for updates

(C) Author(s) (or their employer(s)) 2022. Re-use permitted under CC BY. Published by BMJ.

To cite: Emmons-Bell $\mathrm{S}$, Johnson C, Roth G. Heart Epub ahead of print: [please include Day Month Year]. doi:10.1136/ heartjnl-2021-320131

\section{ABSTRACT}

Studies of the epidemiology of heart failure in the general population can inform assessments of disease burden, research, public health policy and health system care delivery. We performed a systematic review of prevalence, incidence and survival for all available population-representative studies to inform the Global Burden of Disease 2020. We examined populationbased studies published between 1990 and 2020 using structured review methods and database search strings. Studies were sought in which heart failure was defined by clinical diagnosis using structured criteria such as the Framingham or European Society of Cardiology criteria, with studies using alternate case definitions identified for comparison. Study results were extracted with descriptive characteristics including age range, location and case definition. Search strings identified 42360 studies over a 30-year period, of which 790 were selected for full-text review and 125 met criteria for inclusion. 45 sources reported estimates of prevalence, 41 of incidence and 58 of mortality. Prevalence ranged from $0.2 \%$, in a Hong Kong study of hospitalised heart failure patients in 1997 , to $17.7 \%$, in a US study of Medicare beneficiaries aged 65+ from 2002 to 2013. Collapsed estimates of incidence ranged from $0.1 \%$, in the EPidémiologie de I'Insuffisance Cardiaque Avancée en Lorraine (EPICAL) study of acute heart failure in France among those aged $20-80$ years in 1994 , to $4.3 \%$, in a US study of Medicare beneficiaries 65+ from 1994 to 2003. One-year heart failure case fatality ranged from $4 \%$ to $45 \%$ with an average of $33 \%$ overall and $24 \%$ for studies across all adult ages. Diagnostic criteria, case ascertainment strategy and demographic breakdown varied widely between studies. Prevalence, incidence and survival for heart failure varied widely across countries and studies, reflecting a range of study design. Heart failure remains a high prevalence disease among older adults with a high risk of death at 1 year.

\section{INTRODUCTION}

Studies of the epidemiology of heart failure in the general population can inform assessments of disease burden, research, public health policy and health system care delivery. Past investigations of the occurrence of heart failure in the community have most often been performed in the high-income world, however prevalence is projected to rise in low-income and middle-income countries as populations age and the burden of heart failure risk factors such as elevated blood pressure increases in the coming decades. ${ }^{1}$ Heart failure is also likely to confer significant economic burden to individuals and health systems. ${ }^{2}$

The Global Burden of Disease (GBD) study produces comprehensive and comparable estimates of disease burden for 370 causes for 204 countries and territories from 1990 to 2020 , using disease modelling methods. ${ }^{3}$ Regular reviews of published scientific studies are performed to identify data on disease burden, including for heart failure. A focus of this review is the systematic identification of all available data from all countries, with care taken to account for stratification by age and sex, and sought over long timeframes to capture secular trends. Particular attention is paid to variation in disease case definitions and how this may influence observations. Previous reviews by other groups have focused on subtypes of heart failure, specific age groups ${ }^{4} 5$ specific geographic regions ${ }^{6-11}$ or were restricted to prevalence rates only. To date, no review has included prevalence, incidence and rates of survival, covered all geographic regions and included studies from 1990 to the current day. Here, we report the results of such a systematic review identifying data sources to inform the GBD 2020 study estimates of heart failure.

\section{METHODS}

Our review was designed to address specific challenges in the reporting of heart failure burden for the general population. Epidemiological studies of heart failure vary in study design and clinical definition, complicating efforts to produce comparable estimates of disease burden. For example, definitions of heart failure are heterogeneous and include clinical criteria established before non-invasive imaging was widely available, such as the Framingham and the European Society of Cardiology criteria. Some population-based studies also identify heart failure by International Classification of Disease (ICD) or Read codes, which have been shown to vary in some populations from classic clinical criteria level, ${ }^{12}$ and reveal differences between estimates of heart failure prevalence or incidence when applying different clinical scores.

Beginning in 2015, the GBD study has performed an annual systematic review of the literature from 1990 onward to identify all primary data sources with population-representative estimates of the prevalence, incidence or survival rates of heart failure. For this current analysis, we searched PubMed using structured search criteria from 1990 to 2020. Additionally, we included papers sent to us via the network of over 3500 GBD study collaborators or identified in the citations of high-impact studies identified by expert reviewers.

To ensure comparability between data sources, the GBD study defines a gold-standard case definition for each of its 370 reported causes. The case definition for heart failure was that of a clinical diagnosis of heart failure using structured criteria such as the Framingham, European Society of 
Table 1 Studies reporting heart failure prevalence identified in systematic review

\begin{tabular}{|c|c|c|c|c|c|}
\hline & Study & Location & Diagnostic criteria & Setting & Age range \\
\hline \multirow[t]{40}{*}{ High-income } & Cuthbert, 2019 & East Yorkshire, UK & $\begin{array}{l}\text { Read codes for signs and } \\
\text { symptoms }\end{array}$ & Patients from a single practice & \\
\hline & Leibowitz, 2019 & Israel & Signs and symptoms & $\begin{array}{l}\text { Cohort from Jerusalem } \\
\text { Longitudinal Cohort Study }\end{array}$ & Born 1920-1921 \\
\hline & Lindmark, 2019 & Sweden & ICD-10 codes & Electronic medical records & $18+$ \\
\hline & Smeets, 2019 & Belgium & ICPC codes & Patients in participating hospitals & \\
\hline & Cho, 2018 & Republic of Korea & ICD-10 codes & $\begin{array}{l}\text { Health insurance patient sample } \\
\text { (HIRA-NPS) }\end{array}$ & $19+$ \\
\hline & Danielsen, 2017 & Reykjavik, Iceland & AGES-Reykjavikstudy criteria & Random sample from census & Born 1907-1935 \\
\hline & Einarsson, 2017 & Iceland & Ageing Study criteria & Hjartavern's Ageing Study & \\
\hline & Khera, 2017 & USA & ICD-9 codes & $\begin{array}{l}\text { Representative sample of } \\
\text { Medicare records }\end{array}$ & $65+$ \\
\hline & Piccinni, 2017 & Italy & ICD-9 codes & $\begin{array}{l}\text { Patients from participating } \\
\text { hospitals }\end{array}$ & $14+$ \\
\hline & Stork, 2017 & Germany & ICD-10 codes & Insurance records & \\
\hline & Taylor, 2017 & Australia & ICPC codes & $\begin{array}{l}\text { Patients from randomly sampled } \\
\text { practices }\end{array}$ & $45+$ \\
\hline & Lee, 2016 & Republic of Korea & ICD-10 codes & Health insurance records & $19+$ \\
\hline & Tuppin, 2016 & France & ICD-10 codes & Insurance records & \\
\hline & Jiménez-García, 2014 & Madrid, Spain & Chart extraction & Public health system database & \\
\hline & Khan, 2014 & USA & Signs and symptoms & $\begin{array}{l}\text { Random sample of Medicare } \\
\text { beneficiaries }\end{array}$ & $65+$ \\
\hline & Tiller, 2013 & Germany & Signs and symptoms & Cohort study in one community & \\
\hline & Zarrinkoub, 2013 & Stockholm, Sweden & ICD-10 codes & Public health system database & \\
\hline & Mureddu, 2012 & Lazio, Italy & ESC 2005 criteria & Random sample by mail & $65-84$ \\
\hline & Carmona, 2011 & Madrid, Spain & ICPC codes & Electronic medical records & $14+$ \\
\hline & Engelfriet, 2011 & The Netherlands & ICPC codes and E-codes & $\begin{array}{l}\text { Representative general practice } \\
\text { registries }\end{array}$ & \\
\hline & Leibowitz, 2011 & Israel & Signs and symptoms & $\begin{array}{l}\text { Cohort from Jerusalem } \\
\text { Longitudinal Cohort Study }\end{array}$ & Born 1920-1921 \\
\hline & Alehagen, 2009 & Southeast Sweden & Signs and symptoms & Survey of rural municipality & $70-80$ \\
\hline & Anguita Sanchez, 2008 & Spain & Framingham criteria & Registry of participating hospitals & $45+$ \\
\hline & Knox, 2008 & Australia & Signs and symptoms & $\begin{array}{l}\text { Patients in randomly sampled } \\
\text { practices }\end{array}$ & \\
\hline & Ammar, 2007 & Minnesota, USA & Framingham criteria & Random sample of county & $45+$ \\
\hline & Abhayaratna, 2006 & Canberra, Australia & $\begin{array}{l}\text { Self-report verified by record } \\
\text { review }\end{array}$ & $\begin{array}{l}\text { Random sample from electoral } \\
\text { roll }\end{array}$ & $60-85$ \\
\hline & Azevedo, 2006 & Porto, Portugal & Signs and symptoms & Population health survey & $45+$ \\
\hline & Ceia, 2005 & Portugal & ESC 1995 criteria & $\begin{array}{l}\text { Random sampling, primary care } \\
\text { centres }\end{array}$ & $25+$ \\
\hline & Di Bari, 2004 & Dicomano, Italy & ESC 1995 criteria & $\begin{array}{l}\text { Survey of the elderly in small } \\
\text { town }\end{array}$ & $65+$ \\
\hline & McAlister, 2004 & Scotland & $\begin{array}{l}\text { Read codes for signs and } \\
\text { symptoms }\end{array}$ & $\begin{array}{l}\text { Patients from participating } \\
\text { hospitals }\end{array}$ & \\
\hline & Murphy, 2004 & Scotland & $\begin{array}{l}\text { Read codes for signs and } \\
\text { symptoms }\end{array}$ & $\begin{array}{l}\text { Patients from participating } \\
\text { hospitals }\end{array}$ & $18+$ \\
\hline & $\mathrm{Ni}, 2003$ & USA & Self-report & National health statistics & $18+$ \\
\hline & Redfield, 2003 & Minnesota, USA & Chart extraction & Random sample of single county & $45+$ \\
\hline & Ceia, 2002 & Madeira, Portugal & ESC 1995 criteria & $\begin{array}{l}\text { Random sampling, primary care } \\
\text { centres }\end{array}$ & $25+$ \\
\hline & Cortina, 2001 & Asturias, Spain & Signs and symptoms & Random sample from census & $40+$ \\
\hline & Davies, 2001 & West Midlands, England & ESC 1995 criteria & $\begin{array}{l}\text { Sample from primary health } \\
\text { centres }\end{array}$ & $45+$ \\
\hline & Kitzman, 2001 & USA & Signs and symptoms & $\begin{array}{l}\text { Recruitment from participating } \\
\text { field centres }\end{array}$ & $65+$ \\
\hline & Mosterd, 1999 & Rotterdam, The Netherlands & Signs and symptoms & Cohort study of single suburb & $55+$ \\
\hline & Kupari, 1997 & Helsinki, Finland & Signs and symptoms & Random sampling of residents & $\begin{array}{l}\text { Born } 1904,1909 \\
\text { or } 1914\end{array}$ \\
\hline & Kannel, 1991 & USA & Framingham criteria & Framingham study & \\
\hline
\end{tabular}


Table 1 Continued

\begin{tabular}{|c|c|c|c|c|c|}
\hline & Study & Location & Diagnostic criteria & Setting & Age range \\
\hline $\begin{array}{l}\text { Latin America and } \\
\text { Caribbean }\end{array}$ & McSwain, 1999 & Antigua and Barbuda & ICD-10 codes & Patients from referral hospital & \\
\hline $\begin{array}{l}\text { North Africa and Middle } \\
\text { East }\end{array}$ & Agarwal, 2001 & Dhakliya, Oman & Signs and symptoms & Patients in referral hospital & $13+$ \\
\hline \multirow[t]{4}{*}{$\begin{array}{l}\text { Southeast Asia, East Asia } \\
\text { and Oceania }\end{array}$} & Нао, 2019 & China & $\begin{array}{l}\text { Modified ESC } 2016 \text { including } \\
\text { self-report }\end{array}$ & Random sample & $15+$ \\
\hline & Shan, 2014 & China & Signs and symptoms & Random sampling & $60+$ \\
\hline & Dongfeng, 2003 & China & Self-report & Random sample & $35-74$ \\
\hline & Hung, 2000 & China & ICD-9 codes & $\begin{array}{l}\text { Patients in } 11 \text { participating } \\
\text { hospitals }\end{array}$ & \\
\hline
\end{tabular}

AGES, age, gene/environment susceptibility; ESC, European Society of Cardiology; HIRA-NPS, Health Insurance Review and Assessment Service-National Patient Samples; ICD, International Classification of Disease.

Cardiology or Boston criteria. Heart failure identified by ICD, International Classification of Primary Care (ICPC) or Read codes was included if the diagnosis was verified by a physician. This definition captures the American College of Cardiology/American Heart Association stage $\mathrm{C}$ and $\mathrm{D}$, which includes patients with prior or current heart failure, regardless of treatment status.

We screened the titles and abstract of all studies for relevance, the presence of data of interest and study type. In full-text review, we screened for representativeness, diagnostic criteria and epidemiological methodology. We excluded papers that focused only on subpopulations like veterans, data that were not representative and biased geographic selections. Sampled study groups were included as long as sampling resulted in a representative population. We additionally excluded papers without extractable data, such as descriptive reports of registries or heart failure patients, or data at the wrong demographic level, such as estimates of heart failure prevalence stratified by ejection fraction.

We extracted estimates of prevalence, incidence and mortality, defined as case-fatality, with-condition mortality rate, excess mortality rate or standardised mortality ratios. We report first author, publication date, data measure, diagnosis used to identify heart failure, case ascertainment strategy and any demographic restrictions. Additionally, we report estimates of prevalence, incidence and 1 year case fatality, collapsed into the broadest available age and sex categories. When estimates were only available in detailed age or sex categories (such as 10-year age groups or both sexes), we calculated effective sample sizes from reported SE based on the Wilson Score Interval, and then collapsed cases and sample sizes to re-estimate a mean value. Site-years were calculated as the sum of years covered by study, measure and location (eg, Cuthbert et al, 2019, contributes three site-years to the UK as it reports data between 2015 and 2017).

Title/Abstract screening and full-text extraction were performed by separate reviewers. All included papers were reviewed by CJ and GR. We present the full list of studies evaluated in the systematic review in the online supplemental material. Neither patients nor the general public were involved in the design or conduct of this systematic review of the literature.

\section{RESULTS}

The PubMed search returned 42360 studies through 15 May 2020, of which 790 were selected for full-text review and 125 included (online supplemental figure 1). Forty-five sources reported estimates of prevalence, 41 reported estimates of incidence and 58 reported estimates of mortality (tables $1-3$ ). The included studies were published between 1991 and 2019 and represent 51 countries and 911 site-source-years of data.

Design of these studies varied. Seventeen used random sampling or surveys of entire municipalities. Thirty-nine studies used large administrative databases, such as insurance records or state-wide hospital discharges, to identify the study population. Sixteen studies were cohort-based, including the Framingham, Atherosclerosis Risk in Communities Study and Jerusalem Longitudinal Cohort study. Forty-five studies reported patients presenting to participating hospitals, such as a single referral centre or several cooperating sites.

One hundred one of the 125 included studies reported data from high-income regions, which includes Western Europe, North America, Australasia, Southern Latin America and highincome Asia Pacific. Four studies reported data from Central Europe, Eastern Europe and Central Asia; three from Latin America and the Caribbean; two from North Africa and the Middle East; three from South Asia; seven from Southeast Asia, East Asia and Oceania and one from sub-Saharan Africa. The most common locations represented were the USA (23 studies), the UK (8), China (7) and Israel (6). The demographic profile of included patients varied by study (tables 1-3). Some studies restricted to certain age groups, such as patients aged $65+$ years or those born in 1920-1921, while others included patients of all ages. One study surveyed only women.

Figure 1 shows reported values of heart failure prevalence, separated by demographic profile (studies including patients of all ages; all adults, referring to patients aged 18 years and older and older adults, referring to patients 50 and older). When collapsed into the broadest reported age and sex groups, estimates of heart failure prevalence ranged from 0.002 per capita, in a Hong Kong study that enrolled hospitalised heart failure patients and estimated prevalence from the site's catchment area, to 0.18 per capita, in a US study of Medicare beneficiaries aged $65+$ years that captured heart failure with ICD codes (figure 1). The five highest prevalence values reported were from studies focusing on patients aged 50+ years. Among studies limited to older adults, the average of reported prevalence values was 8.3\%. Among studies limited to all adults, average reported prevalence was $3.4 \%$. Among studies enrolling patients of all ages, average reported prevalence was $1.3 \%$.

The most common locations reporting prevalence were the USA (five studies), Spain (four studies), Australia (three studies), Portugal (three studies), China (three studies) and Sweden (three studies). In prevalence studies, heart failure was diagnosed by signs and symptoms (including Framingham, ESC and Boston 
Table 2 Studies reporting heart failure incidence identified in systematic review

\begin{tabular}{|c|c|c|c|c|c|}
\hline & Study & Location & Diagnostic criteria & Setting & Age range \\
\hline $\begin{array}{l}\text { Central Europe, Eastern } \\
\text { Europe and Central } \\
\text { Asia }\end{array}$ & Rywik, 1999 & Poland & ICD-9 codes & National healthcare records & \\
\hline \multirow[t]{37}{*}{ High-income } & Huusko, 2019 & Southwest Finland & ICD-10 codes & Electronic medical records & $18+$ \\
\hline & Li, 2019 & USA & Signs and symptoms & $\begin{array}{l}\text { Sample from existing population-based } \\
\text { studies }\end{array}$ & $40+$ \\
\hline & Lindmark, 2019 & Sweden & ICD-10 codes & Electronic medical records & $18+$ \\
\hline & Magnussen, 2019 & Western Europe & $\begin{array}{l}\text { Self-report, signs and symptoms or } \\
\text { ICD-10 }\end{array}$ & Patients in four cohort studies & \\
\hline & Uijl, 2019 & The Netherlands & ICD-9 and ICD-10 codes & $\begin{array}{l}\text { Two cohort studies (MORGEN, } \\
\text { Prospect) }\end{array}$ & \\
\hline & Conrad, 2018 & UK & ICD-10 codes & Electronic medical records & $16+$ \\
\hline & Hinton, 2018 & England & Read codes for signs and symptoms & Patients in 164 participating centres & $18+$ \\
\hline & Shah, 2018 & Massachusetts, USA & Framingham criteria & Framingham offspring study & \\
\hline & Tsao, 2018 & USA & Framingham criteria & $\begin{array}{l}\text { Framingham original and offspring } \\
\text { study }\end{array}$ & \\
\hline & Einarsson, 2017 & Iceland & Ageing Study criteria & Hjartavern's Ageing Study & \\
\hline & Khera, 2017 & USA & ICD-9 codes & $\begin{array}{l}\text { Representative sample of Medicare } \\
\text { records }\end{array}$ & $65+$ \\
\hline & Piccinni, 2017 & Italy & ICD-9 codes & Patients from participating hospitals & $14+$ \\
\hline & Stork, 2017 & Germany & ICD-10 codes & Insurance records & \\
\hline & Nayor, 2016 & Massachusetts, USA & Framingham criteria & Framingham offspring study & \\
\hline & Sangaralingham, 2016 & USA & ICD-9 codes & Commercial insurance database & \\
\hline & Ohlmeier, 2015 & Germany & ICD-10 codes & Insurance records & \\
\hline & Barasa, 2014 & Sweden & ICD-9 and ICD-10 codes & Hospital discharges, death registry & $18-84$ \\
\hline & Borne, 2014 & Malmo, Sweden & ICD-9 and ICD-10 codes & Cohort study (MDC) & $\begin{array}{l}\text { Born 1923- } \\
1950\end{array}$ \\
\hline & Corrao, 2014 & Lombardy, Italy & ICD-9 codes & Health services database & \\
\hline & Khan, 2014 & USA & Signs and symptoms & $\begin{array}{l}\text { Random sample of Medicare } \\
\text { beneficiaries }\end{array}$ & $65+$ \\
\hline & Rautiainen, 2013 & Sweden & ICD-10 codes & Cohort study of two counties & $\begin{array}{l}\text { Women } \\
\text { Born 1914- } \\
1948\end{array}$ \\
\hline & Shah, 2013 & USA & Cardiovascular Health Study criteria & $\begin{array}{l}\text { Cohort study in six communities } \\
\text { (MESA) }\end{array}$ & \\
\hline & Zarrinkoub, 2013 & Stockholm, Sweden & ICD-10 codes & Public health system database & \\
\hline & Wasywich, 2010 & New Zealand & ICD-9 codes & Public health system database & $18+$ \\
\hline & Curtis, 2008 & USA & ICD-9 codes & $\begin{array}{l}\text { Representative sample of Medicare } \\
\text { records }\end{array}$ & $65+$ \\
\hline & Loehr, 2008 & USA & ICD- 9 codes & Population-based cohort (ARIC) & $45-64$ \\
\hline & van Jaarsveld, 2006 & Northern Netherlands & ICPC codes & Sample from participating GPs & $57+$ \\
\hline & de Giuli, 2005 & UK & Chart extraction & Sample from general practice database & $45+$ \\
\hline & Bleumink, 2004 & $\begin{array}{l}\text { Rotterdam, The } \\
\text { Netherlands }\end{array}$ & $\begin{array}{l}\text { European Society of Cardiology } 2001 \\
\text { criteria }\end{array}$ & Cohort study of single suburb & $55+$ \\
\hline & Lee, 2004 & Canada & ICD-9 codes & Hospital discharges, death registry & 20-105 \\
\hline & McAlister, 2004 & Scotland & Read codes for signs and symptoms & Patients from participating hospitals & \\
\hline & Murphy, 2004 & Scotland & Read codes for signs and symptoms & Patients from participating hospitals & $18+$ \\
\hline & Fox, 2001 & South London, UK & $\begin{array}{l}\text { European Society of Cardiology } 1995 \\
\text { criteria }\end{array}$ & Registry of participating practices & \\
\hline & Senni, 1999 & Minnesota, USA & Framingham criteria & Random sample of single county & \\
\hline & Zannad, 1999 & Lorraine, France & Signs and symptoms & Patients from participating hospitals & $20-80$ \\
\hline & Remes, 1992 & Eastern Finland & Boston criteria & Patients from participating hospitals & $45-74$ \\
\hline & Kannel, 1991 & USA & Framingham criteria & Framingham study & \\
\hline $\begin{array}{l}\text { North Africa and } \\
\text { Middle East }\end{array}$ & Al Suwaidi, 2004 & Qatar & Framingham criteria & Patients in referral hospital & \\
\hline Southeast Asia, East & Tseng, 2011 & Taiwan (Province of China) & ICD-9 codes & Random sample of insurance registrar & $20+$ \\
\hline Asia and Oceania & Hung, 2000 & China & ICD-9 codes & Patients in 11 participating hospitals & \\
\hline
\end{tabular}


Table 3 Studies reporting heart failure mortality identified in systematic review

\begin{tabular}{|c|c|c|c|c|c|}
\hline & Study & Location & Diagnostic criteria & Setting & Age range \\
\hline \multirow{3}{*}{$\begin{array}{l}\text { Central Europe, Eastern Europe and } \\
\text { Central Asia }\end{array}$} & Kaplon-Cieslicka, 2016 & Poland & ESC 2012 criteria & Polish cohort of ESC registry & $18+$ \\
\hline & Ozieranski, 2016 & Poland & ESC 2012 criteria & Polish cohort of ESC registry & $18+$ \\
\hline & Parenica, 2013 & Czechia & Signs and symptoms & Patients from participating hospitals & \\
\hline \multirow[t]{46}{*}{ High-income } & Canepa, 2019 & Italy & Signs and symptoms & Randomised nested trial (GISSI-HF) & \\
\hline & Chen, 2019 & Sweden & ICD-10 codes & Swedish Heart Failure Registry & \\
\hline & Stork, 2017 & Germany & ICD-10 codes & Insurance records & \\
\hline & Nakano, 2016 & Denmark & ICD-10 codes & National healthcare records (DHFR) & $18+$ \\
\hline & Schmidt, 2016 & Denmark & ICD-8 and ICD-10 codes & Hospital discharges, death registry & \\
\hline & Staszewsky, 2016 & Lombardy, Italy & Chart extraction & Linked healthcare databases & \\
\hline & Atzema, 2015 & Canada & ICD-10 codes & $\begin{array}{l}\text { Citizen registrar, healthcare } \\
\text { databases }\end{array}$ & \\
\hline & Berkovitch, 2015 & Israel & Signs and symptoms & Survey of patients in 25 hospitals & \\
\hline & Coles, 2015 & Massachusetts, USA & Framingham criteria & Patients in 11 contributing centres & $18+$ \\
\hline & Ohlmeier, 2015 & Germany & ICD-10 codes & Insurance records & \\
\hline & Vanhercke, 2015 & Belgium & ESC 2015 criteria & Patients in participating hospitals & $18+$ \\
\hline & Barasa, 2014 & Sweden & ICD-9 and ICD-10 codes & Hospital discharges, death registry & $18-84$ \\
\hline & Corrao, 2014 & Lombardy, Italy & ICD-9 codes & Health services database & \\
\hline & Sartipy, 2014 & Sweden & ICD-10 codes & Swedish Heart Failure Registry & \\
\hline & Tuppin, 2014 & France & ICD-10 codes & Insurance records & \\
\hline & Chamberlain, 2013 & Minnesota, USA & Framingham criteria & Electronic medical records & \\
\hline & Hoekstra, 2013 & The Netherlands & ESC 2008 criteria & Patients in 17 participating hospitals & $18+$ \\
\hline & Lassus, 2013 & Finland & Signs and symptoms & Patients from participating hospitals & \\
\hline & Maison, 2013 & France & WHO classification & Patients from participating hospitals & \\
\hline & McAlister, 2013 & Alberta, Canada & ICD-9 and ICD-10 codes & Linked healthcare registries & $18+$ \\
\hline & McManus, 2013 & Massachusetts, USA & Framingham criteria & Patients from participating hospitals & $18+$ \\
\hline & Nakano, 2013 & Denmark & ICD-10 codes & National healthcare records (DHFR) & $18+$ \\
\hline & Oster, 2013 & Israel & Signs and symptoms & Patients from participating hospitals & \\
\hline & Chen, 2011 & USA & ICD-9 codes & $\begin{array}{l}\text { Review of Medicare claims data } \\
\text { (CMS) }\end{array}$ & $65+$ \\
\hline & Ezekowitz, 2011 & Alberta, Canada & ICD-9 and ICD-10 codes & Linked healthcare registries & \\
\hline & Gamble, 2011 & Alberta, Canada & ICD-9 and ICD-10 codes & Linked healthcare registries & \\
\hline & Goda, 2010 & Tokyo, Japan & Signs and symptoms & Patients in referral hospital & \\
\hline & Novack, 2010 & Israel & ICD-9 codes & Patients from participating hospitals & \\
\hline & Tribouilloy, 2010 & France & Framingham and ESC 1995 criteria & Patients from participating hospitals & $20+$ \\
\hline & Wasywich, 2010 & New Zealand & ICD-9 codes & Public health system database & $18+$ \\
\hline & Jhund, 2009 & Scotland & ICD-9 and ICD-10 codes & Hospital discharges, death registry & \\
\hline & Amsalem, 2008 & Israel & Signs and symptoms & $\begin{array}{l}\text { Heart Failure Survey in Israel } \\
\text { database }\end{array}$ & \\
\hline & Ko, 2008 & Ontario, Canada & Framingham criteria & Records from participating hospitals & $20-105$ \\
\hline & Shiba, 2008 & Japan & Signs and symptoms & $\begin{array}{l}\text { Patients in participating hospitals } \\
\text { (CHART-1) }\end{array}$ & $18+$ \\
\hline & Ammar, 2007 & Minnesota, USA & Framingham criteria & Random sample of county & $45+$ \\
\hline & Rathore, 2006 & USA & ICD-9 codes & Sample of Medicare beneficiaries & $65+$ \\
\hline & van Jaarsveld, 2006 & Northern Netherlands & ICPC codes & Sample from participating GPs & $57+$ \\
\hline & Bleumink, 2004 & Rotterdam, The Netherlands & ESC 2001 criteria & Cohort study of single suburb & $55+$ \\
\hline & Lee, 2004 & Ontario, Canada & ICD-9 codes & Hospital discharges, death registry & $65+$ \\
\hline & Shahar, 2004 & Minnesota, USA & ICD-9 codes & Patients in participating counties & $35-84$ \\
\hline & Sosin, 2004 & UK & ESC 2001 criteria & Patients in participating hospitals & \\
\hline & Lee, 2003 & Ontario, Canada & ICD-9 codes & Patients from participating hospitals & \\
\hline & Cowie, 2000 & West London, England & Adapted ESC 1995 criteria & Patients in district hospital & \\
\hline & Heller, 2000 & South Wales, Australia & ICD-9 and ICD-10 codes & $\begin{array}{l}\text { Patients in } 22 \text { hospitals (Heart and } \\
\text { Stroke Register) }\end{array}$ & \\
\hline & Tsuchihashi, 2000 & Japan & Framingham criteria & Patients from participating hospitals & \\
\hline & Alexander, 1999 & California, USA & ICD-9 codes & Review of CA hospital discharges & \\
\hline \multirow[t]{3}{*}{ Latin America and Caribbean } & Gioli-Pereira, 2019 & Sao Paulo, Brazil & Signs and symptoms & Patients from a single practice & $18-80$ \\
\hline & Lalljie, 2007 & Jamaica & Framingham criteria & Patients from a single practice & \\
\hline & AHRI, 2013 & India & ESC 2012 criteria & Patients from participating hospitals & \\
\hline \multirow[t]{2}{*}{ South Asia } & SCTIMST, 2006 & India & ESC 1995 criteria & Patients from participating hospitals & \\
\hline & SCTIMST, 2001 & India & ESC 1995 criteria & Patients from participating hospitals & \\
\hline \multirow{3}{*}{$\begin{array}{l}\text { Southeast Asia, East Asia and } \\
\text { Oceania }\end{array}$} & Lyu, 2019 & China & Boston criteria & Patients from participating hospitals & $18+$ \\
\hline & Hai, 2016 & $\begin{array}{l}\text { Hong Kong Special Administrative } \\
\text { Region of China }\end{array}$ & Framingham criteria & Patients from a single hospital & $18+$ \\
\hline & Hung, 2000 & China & ICD-9 codes & Patients in 11 participating hospitals & \\
\hline Sub-Saharan Africa & Makubi, 2016 & United Republic of Tanzania & Framingham criteria & Patients from referral hospital & $18+$ \\
\hline
\end{tabular}




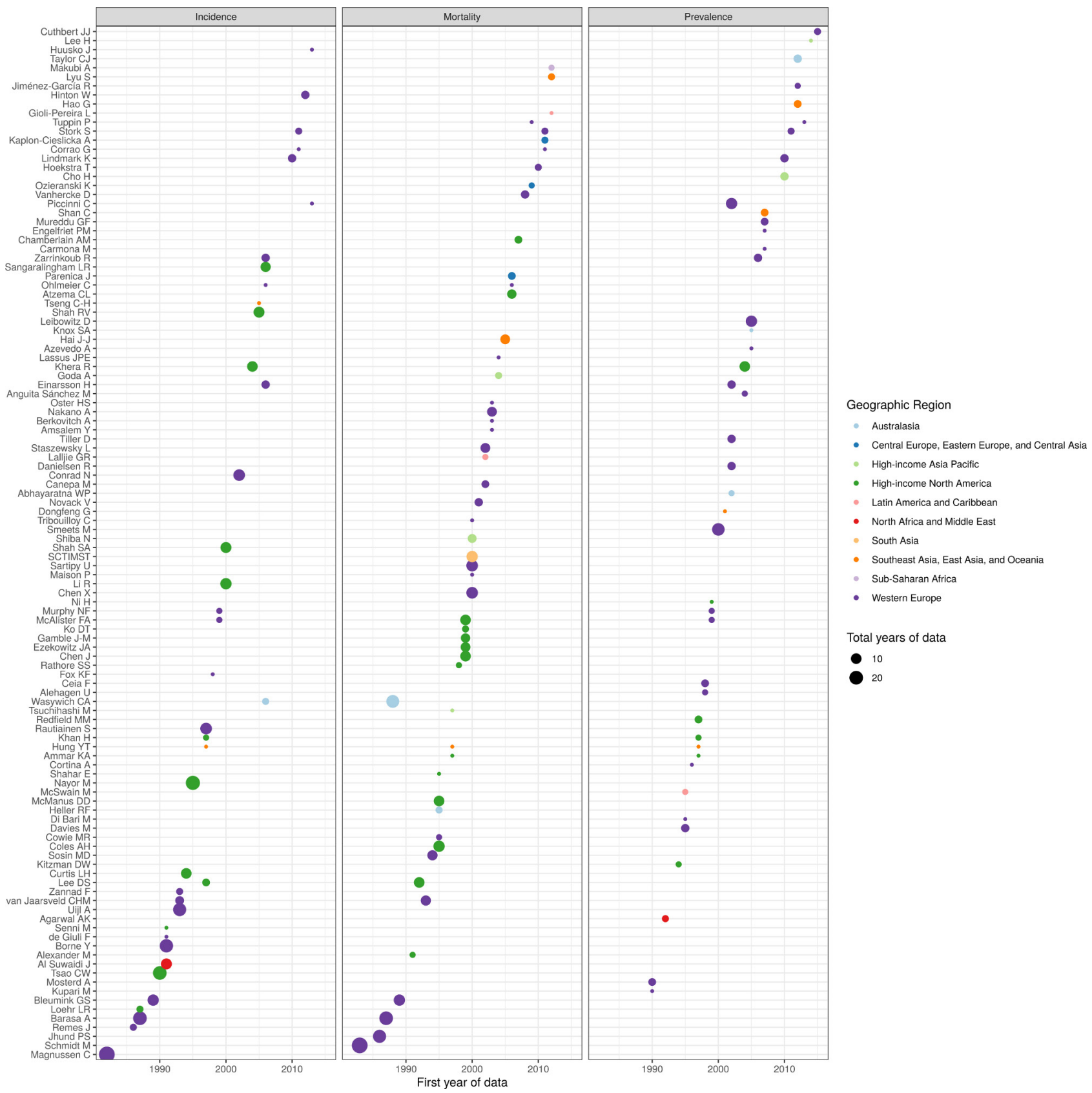

Figure 1 Reported prevalence of heart failure in 45 studies identified in systematic review.

criteria, and chart review) in 26 studies, and by ICD, ICPC or Read codes in 17 studies (table 1). Sampling techniques for these studies included random sampling from primary care centres, random sampling from official census records, review of electronic medical records and medical surveys administrated to entire towns or populations.

Figure 2 shows reported values of heart failure incidence, separated by demographic profile. Reported estimates of heart failure incidence ranged from 100/100 000 person-years, in the French EPICAL study of acute heart failure in those aged $20-80$ years, to $4300 / 100000$ person-years in a US study of Medicare beneficiaries $65+$ identifying heart failure with ICD codes (figure 2). Among studies limited to older adults, the average of reported incidence values was 1600/100 000 person-years. Among studies limited to all adults, average incidence was 840/100 000 personyears. Among studies enrolling patients of all ages, average reported incidence was 460/100 000 person-years.
Common locations reporting heart failure incidence were the USA (12 studies), the UK (6), Sweden (4) and the Netherlands (3). Heart failure incidence was diagnosed by signs and symptoms (including Framingham, ESC and Boston criteria, and chart review) in 16 studies, and by ICD, ICPC or Read codes in 25 studies (table 2). In these studies, sampling techniques included random sample of insurance registrar, hospital and death registry, population-based cohort and analysis of linked public health systems databases.

Figure 3 shows reported values of 1-year heart failure case fatality, separated by demographic profile. Reported estimates of 1 -year case fatality ranged from $4 \%$, in a study that randomly sampled Minnesota residents, to 45\%, in a 1994 study of acute heart failure admissions in Birmingham (figure 3). Among studies limited to older adults, the average of reported 1-year case fatality values was $33 \%$. Among studies limited to all adults, average reported 1-year case fatality was $24 \%$. Among studies 


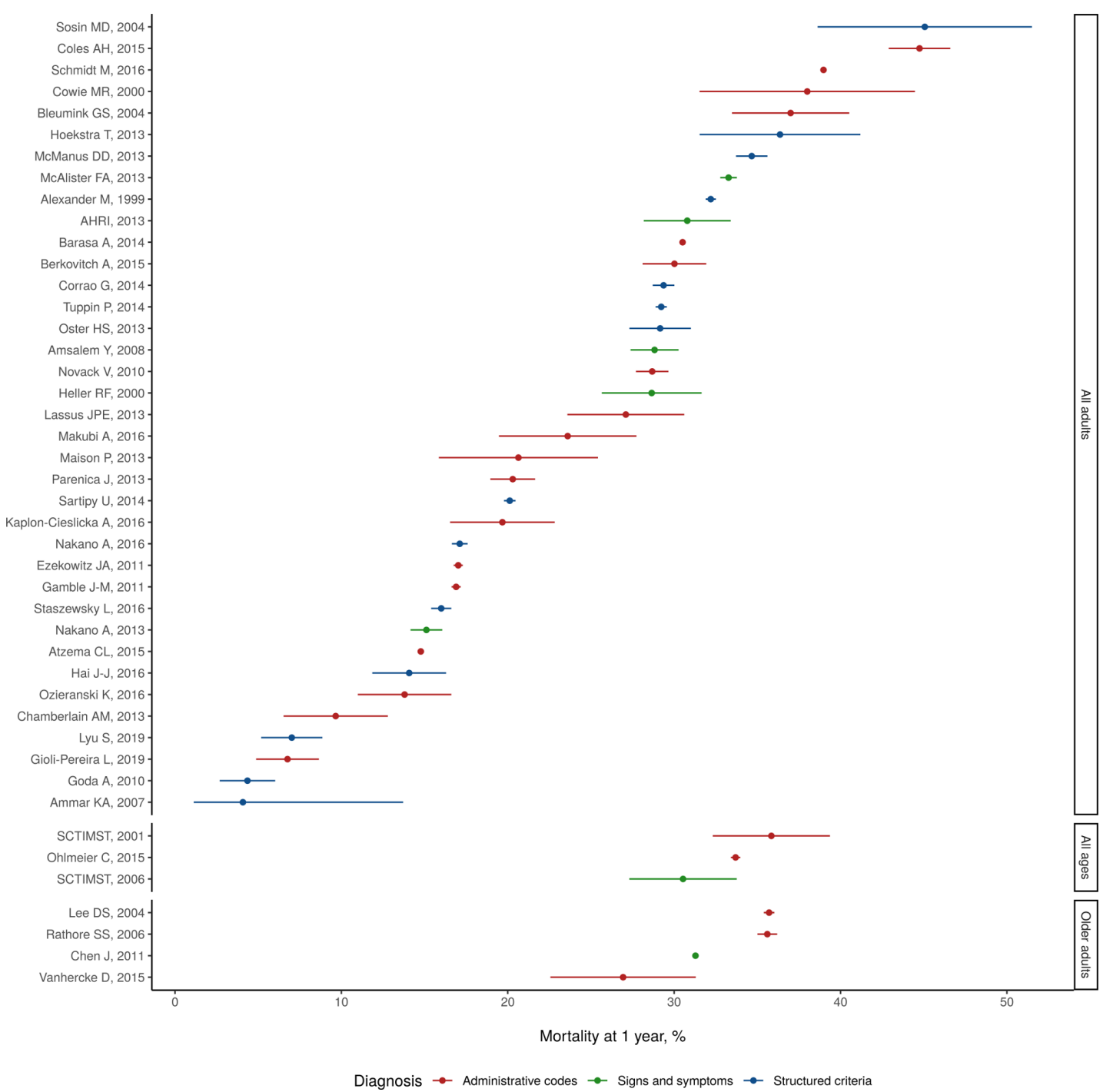

Figure 2 Reported incidence of heart failure in 41 studies identified in systematic review.

enrolling patients of all ages, average reported 1-year case fatality was $33 \%$.

Common locations reporting heart failure case fatality were the USA (seven studies), Canada (6), India (5) and Israel (4). One-year heart failure case fatality was diagnosed by signs and symptoms (including Framingham, ESC and Boston criteria, and chart review) in 25 studies, and by ICD, ICPC or Read codes in 23 studies (table 3). In these studies, sampling techniques included random sample of primary or specialty care centres, review of electronic medical records or insurance records and medical surveys administrated to entire towns or populations.

Studies from 23 countries report estimates of heart failure prevalence or incidence (online supplemental figure 2). Additionally, studies from 23 countries report estimates of heart failure mortality (online supplemental figure 3). Figure 4 shows the number of data-years contributed by each study, coloured by geographic region. Of 911 total site-years of data, 817 were from high-income locations (figure 4). Studies varied in case ascertainment criteria, heart failure diagnosis type, epidemiological design and demographic breakdown. Several papers reported on long-running studies like Framingham or the AGES-Reykjavik study, while others were estimates from a single year or site. Many studies included all patients managed for heart failure by participating hospitals, general practitioners or clinics; these often provided an estimate of catchment area to calculate prevalence or incidence. Some studies used large insurance databases or national administrative healthcare records to identify heart failure patients. Still others were reports of community-based surveys that invited patients to conduct a health screen and heart failure assessment. Many studies did not report specific diagnostic criteria beyond physician diagnosis and are noted as 'signs and symptoms' in the table. The age and sex breakdown of heart failure cases and sample sizes differed by study and were not always reported in granular detail; aggregated estimates reflect this variation.

\section{DISCUSSION}

Our prospective systematic review identified 125 studies reporting prevalence, incidence or mortality of heart failure, synthesising the landscape of epidemiological research on heart failure. Data reported in these studies will inform the GBD 2020 study, help elucidate the global epidemiology of heart failure and guide resources, research and interventions.

These studies describe a prevalence and incidence of heart failure that varies widely across locations. Much of the observed variation may reflect true changes in the age-specific burden of heart failure within specific populations. Our results suggest 


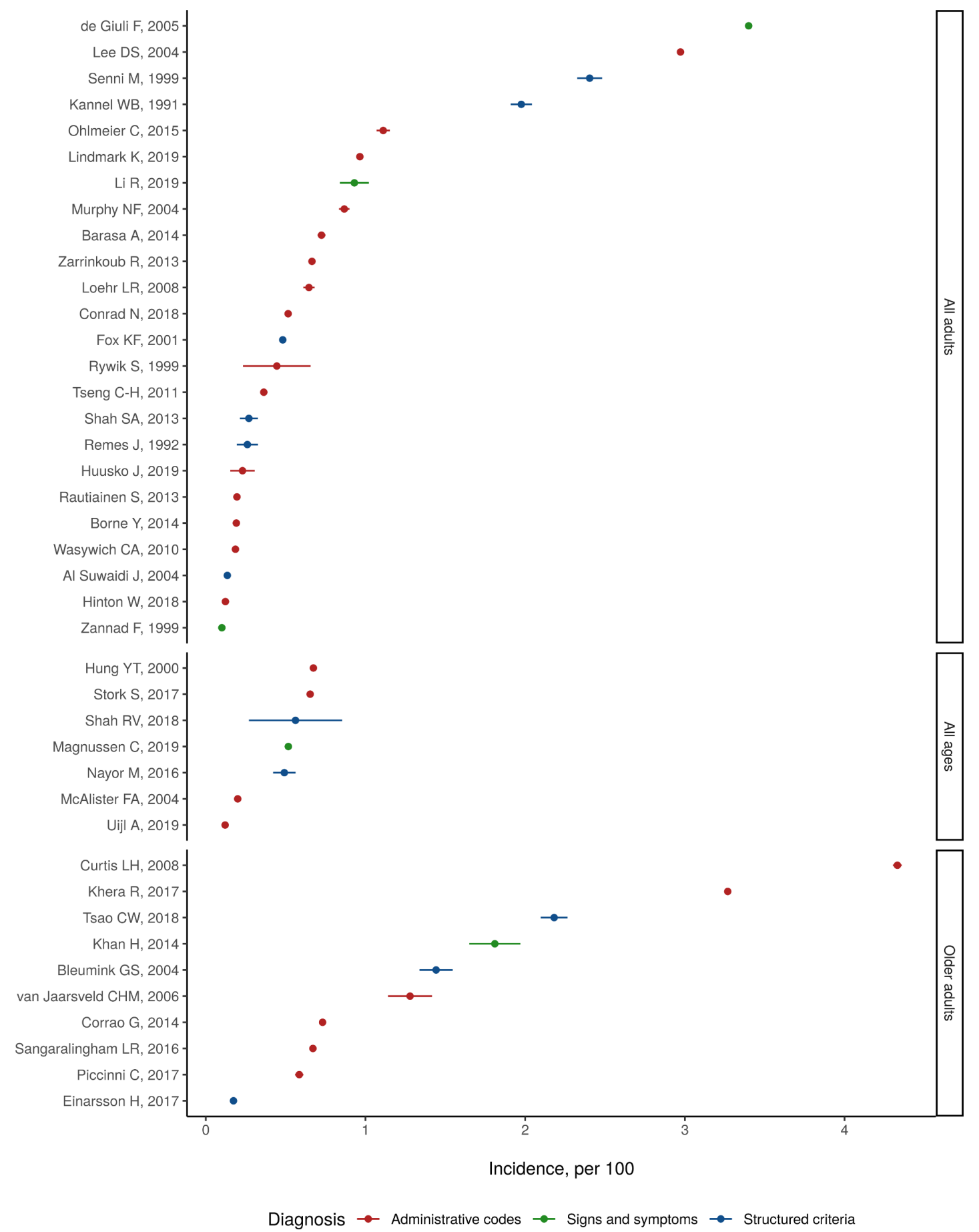

Figure 3 Reported 1-year case fatality of heart failure in 44 studies identified in systematic review.

that differences in study design and case ascertainment strategy may also contribute to the observed heterogeneity. Heart failure remains a condition frequently identified when patients develop acute symptoms and, at times, are clinically unstable. Especially relevant are differences in diagnostic criteria, whose sensitivity and specificities reflect clinical judgement across diverse and complex settings such as emergency departments and primary care offices. While some studies apply research-grade enrolment protocols in these settings or even extend surveillance to households, many remain simple counts of acute decompensation of heart failure. As technologies for non-invasive evaluation of heart failure improve, there is a need to shift studies of heart failure epidemiology from case identification based on physical examination and cardiac auscultation to a standardised application of rapid, inexpensive and robust laboratory and echocardiographic criteria.

While the GBD has developed methodology to estimate and correct for systematic bias between case definitions, ${ }^{13}{ }^{14}$ alignment of standards for epidemiological studies of heart failure would improve the comparability between studies and reduce the need for statistical bias correction. National and international societies could help align criteria for epidemiological purposes similar to standardised reporting used for cardiac arrest and myocardial infarction, and standard data collection methods could be adopted for health surveys for non-communicable diseases. Additionally, this review presents collapsed estimates, 


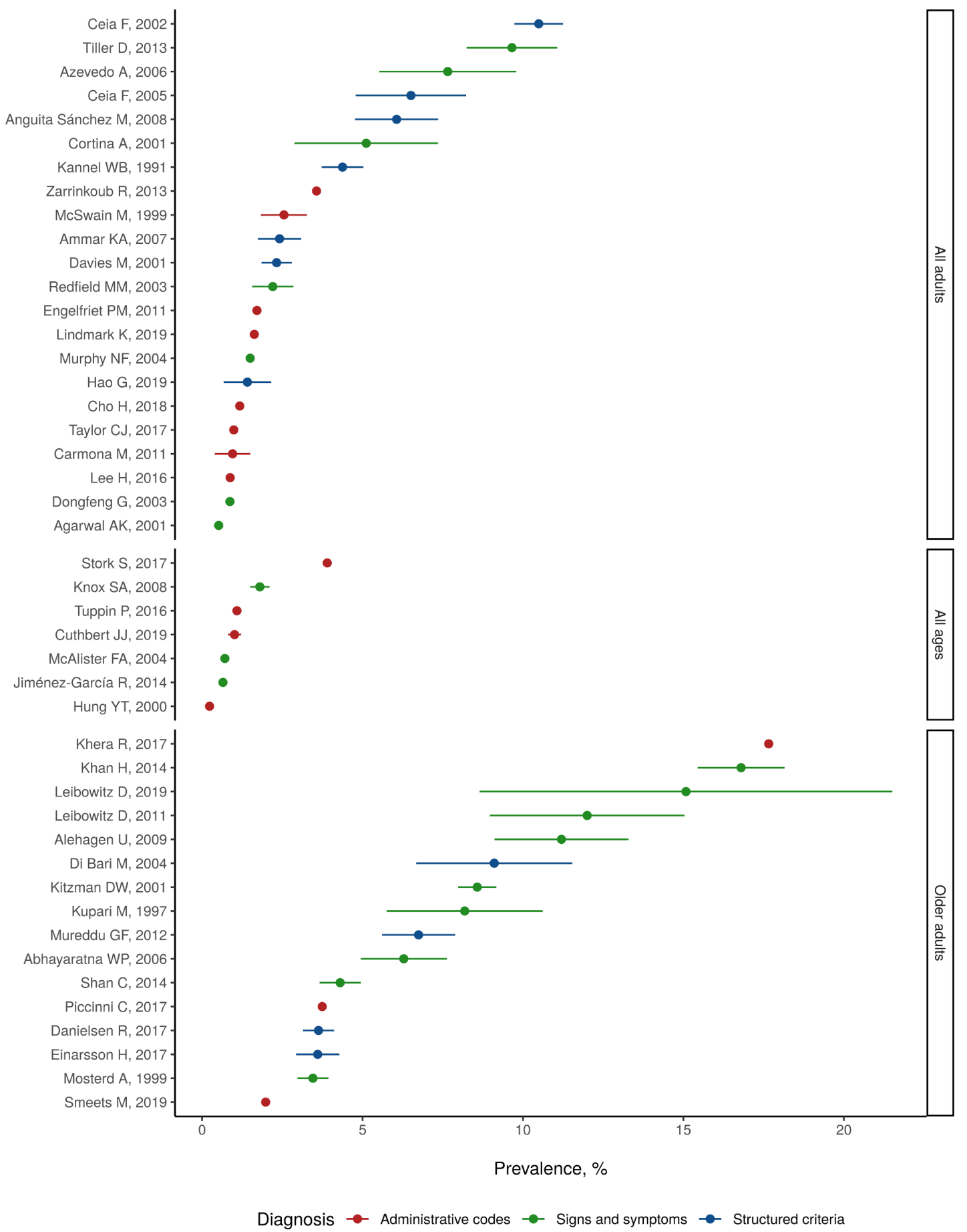

Figure 4 Data coverage by year, measure and geographic region.

not ones standardised to a reference population, so heterogeneity in population structures remain present in the summarised estimates.

High-quality data from more geographical regions is also necessary to understand global patterns and the manner in which diverse pathophysiological aetiologies may affect patterns of heart failure. Although this review identified data from 51 countries, only 11 countries were outside of the high-income world: the Czech Republic, Poland, Antigua and Barbuda, Jamaica, Brazil, Oman, Qatar, India, China, Taiwan and Tanzania. Together, only 94 of 911 site-years of data were outside of the high-income world. Given this, covariates and statistical models are necessary to make estimates of the burden of heart failure in countries or regions where there is limited data. Further investments in data collection and population-based surveys in such locations would improve our understanding of global patterns. Additional data are also needed to better understand the causal pathways by which a wide variety of cardiovascular and other diseases drive the incidence of heart failure, and how these conditions vary across regions in their overall contribution to heart failure prevalence.

\section{CONCLUSION}

Prevalence, incidence and survival for heart failure varied widely across countries and studies, reflecting a range of study design. 
Heart failure remains a high prevalence disease among older adults with a high risk of death at 1 year. This review synthesises all available published estimates of heart failure burden. Future efforts will include the use of geospatial statistical models to produce estimates of global disease burden due to heart failure. Given its place as a common final pathway for a broad set of conditions, an improved understanding of heart failure in the general population would be useful to guide research, resource allocation and policy, and to inform larger efforts to reduce the burden of non-communicable diseases.

Contributors SE-B screened the titles and abstracts of all papers, designed tables and figures, and authored the manuscript. CJ and GR planned the study, oversaw literature screening, reviewed all included papers, and revised the manuscript. GR managed the overall content and is the guarantor of this study. All authors critically reviewed the manuscript for intellectual content and integrity.

Funding This research was supported by the Cardiovascular Medical Research and Education Fund and the Bill and Melinda Gates Foundation. The funders had no role in study design.

Competing interests None declared.

Patient consent for publication Not applicable.

Ethics approval The University of Washington IRB Committee approved the Global Burden of Diseases, Injuries and Risk Factors Study (STUDY00009060).

Provenance and peer review Not commissioned; externally peer reviewed.

Supplemental material This content has been supplied by the author(s). It has not been vetted by BMJ Publishing Group Limited (BMJ) and may not have been peer-reviewed. Any opinions or recommendations discussed are solely those of the author(s) and are not endorsed by BMJ. BMJ disclaims all liability and responsibility arising from any reliance placed on the content. Where the content includes any translated material, BMJ does not warrant the accuracy and reliability of the translations (including but not limited to local regulations, clinical guidelines, terminology, drug names and drug dosages), and is not responsible for any error and/or omissions arising from translation and adaptation or otherwise.

Open access This is an open access article distributed in accordance with the Creative Commons Attribution 4.0 Unported (CC BY 4.0) license, which permits others to copy, redistribute, remix, transform and build upon this work for any purpose, provided the original work is properly cited, a link to the licence is given, and indication of whether changes were made. See: https://creativecommons.org/ licenses/by/4.0/.

ORCID iD

Gregory Roth http://orcid.org/0000-0002-8355-9146

\section{REFERENCES}

1 Yusuf S, Reddy S, Ounpuu S, et al. Global burden of cardiovascular diseases: Part I: general considerations, the epidemiologic transition, risk factors, and impact of urbanization. Circulation 2001;104:2746-53.

2 Cook C, Cole G, Asaria P, et al. The annual global economic burden of heart failure. Int J Cardiol 2014;171:368-76.

3 GBD 2019 Diseases and Injuries Collaborators. Global burden of 369 diseases and injuries in 204 countries and territories, 1990-2019: a systematic analysis for the global burden of disease study 2019. Lancet 2020;396:1204-22.

4 Shaddy RE, George AT, Jaecklin T, et al. Systematic literature review on the incidence and prevalence of heart failure in children and adolescents. Pediatr Cardiol 2018;39:415-36.

5 van Riet EES, Hoes AW, Wagenaar KP, et al. Epidemiology of heart failure: the prevalence of heart failure and ventricular dysfunction in older adults over time. A systematic review. Eur J Heart Fail 2016;18:242-52.

6 Sakata Y, Shimokawa H. Epidemiology of heart failure in Asia. Circ J 2013;77:2209-17.

7 Sahle BW, Owen AJ, Mutowo MP, et al. Prevalence of heart failure in Australia: a systematic review. BMC Cardiovasc Disord 2016;16:32.

8 Woods JA, Katzenellenbogen JM, Davidson PM, et al. Heart failure among Indigenous Australians: a systematic review. BMC Cardiovasc Disord 2012;12:99.

9 Callender T, Woodward M, Roth G, et al. Heart failure care in low- and middle-income countries: a systematic review and meta-analysis. PLoS Med 2014;11:e1001699.

10 Ciapponi A, Alcaraz A, Calderón M, et al. Burden of heart failure in Latin America: a systematic review and meta-analysis. Rev Esp Cardiol 2016;69:1051-60.

11 Agbor VN, Essouma M, Ntusi NAB, et al. Heart failure in sub-Saharan Africa: a contemporaneous systematic review and meta-analysis. Int J Cardiol 2018;257:207-15.

12 Delekta J, Hansen SM, AlZuhairi KS, et al. The validity of the diagnosis of heart failure (I50.0-I50.9) in the Danish national patient register. Dan Med J 2018;65:A5470.

13 Zheng P, Barber R, Sorensen RJD. Trimmed constrained mixed effects models: formulations and algorithms. J Comput Graph Stat 2021;0:1-13.

14 Murray CJL, Aravkin AY, Zheng P, et al. Global burden of 87 risk factors in 204 countries and territories, 1990-2019: a systematic analysis for the global burden of disease study 2019. Lancet 2020;396:1223-49. 